\title{
The tidal effects on the lithium abundance of binary systems with giant component ${ }^{\star}$
}

\author{
J. M. $\operatorname{Costa}^{1}$, L. da Silva ${ }^{2}$, J. D. do Nascimento Jr. ${ }^{1}$, and J. R. De Medeiros ${ }^{1}$ \\ 1 Departamento de Física, Universidade Federal do Rio Grande do Norte, 59072-970 Natal, R.N., Brazil \\ 2 Observatório Nacional, Rio de Janeiro, RJ, Brazil
}

Received 21 July 2001 / Accepted 22 November 2001

\begin{abstract}
We analyse the behavior of lithium abundance as a function of effective temperature, projected rotational velocity, orbital period and eccentricity for a sample of 68 binary systems with giant component and orbital period ranging from about 10 to 6400 days. For these binary systems the $\mathrm{Li}$ abundances show a gradual decrease with temperature, paralleling the well established result for single giants. We have also observed a dependence of lithium content on rotation. Binary systems with moderate to high rotation present also moderate to high Li content. This study shows also that synchronized binary systems with giant component seem to retain more of their original lithium than the unsynchronized systems. For orbital periods lower than 100 to 250 days, typically the period of synchronization for this kind of binary systems, lithium depleted stars seems to be unusual. The suggestion is made that there is an "inhibited zone" in which synchronized binary systems with giant component having lithium abundance lower than a threshold level should be unusual.
\end{abstract}

Key words. stars: binaries - stars: abundances - stars: evolution - stars: interiors - stars: late-type

\section{Introduction}

A correlation between the lithium content and the amount of angular momentum lost by late-type stars is predicted by different authors (e.g. Pinsonneaut et al. 1989, 1990; Zanh 1992, 1994). Such a prediction is indeed confirmed by observations. For unevolved late-type stars in young open clusters such as $\alpha$-Persei and in Pleiades there is a clear dependence of lithium abundance on rotational velocity, in the sense that the fastest rotators are generally stars with enhanced lithium content (Garcia López et al. 1994; Randich et al. 1998). For subgiant stars the same behavior was found by do Nascimento et al. (2000), Randich et al. (1999) and De Medeiros et al. (1997), whereas Barrado et al. (1998) confirmed such a correlation for chromospheric active binary systems. More recently De Medeiros et al. (2000) have found the same behavior for a dependence of lithium content on rotation in giant stars, showing that single giants with high lithium content present also high rotation rate. Such a link between lithium depletion and angular momentum loss is also predicted for binary systems with late-type components. In close enough binary systems viscous dissipation of time-dependent tidal effects should produce a synchronization between rotation and stellar orbital motion as well as the circularization of the orbit of the system (e.g. Zahn 1977). Thus, the angular

\footnotetext{
Send offprint requests to: J. M. Costa,

e-mail: damata@dfte.ufrn.br

* Based on observations collected at ESO, La Silla.
}

momentum which is lost via stellar winds is drawn from the orbital motion, but with the result that the stars are spinning up, differently of single stars which are spinning down. In this context, for a given spectral type, a binary component will have a rotation enhanced in relation to its single counterparts and to those components of binary systems with orbital period larger than the critical period for synchronization. Hence, if the lithium depletion is related to the loss of angular momentum, the surface abundance of this element in synchronized binary systems should be less depleted than that of its single counterparts and that of nonsynchronized binary systems. In fact, Zahn (1994) has shown that late-type binary systems of short enough orbital period retain more of their original Li than their single counterparts. This author has found that such a period is typically below 8 days for solar-type stars of population I and below 6 days for halo stars. The same trend was found by Spite et al. (1994) for old disk and halo stars. This inhibition of lithium depletion was also observed in subgiant stars of population I (De Medeiros et al. 1997; Randich et al. 1999), whereas Barrado et al. (1998) have found a similar result for giant components of chromospheric active binary systems. In the present work we analyse the effects of binarity on the lithium abundances of binary systems with evolved component of luminosity class III, typically F, G and $\mathrm{K}$ type giants, on the basis of high precision spectroscopic observations. 


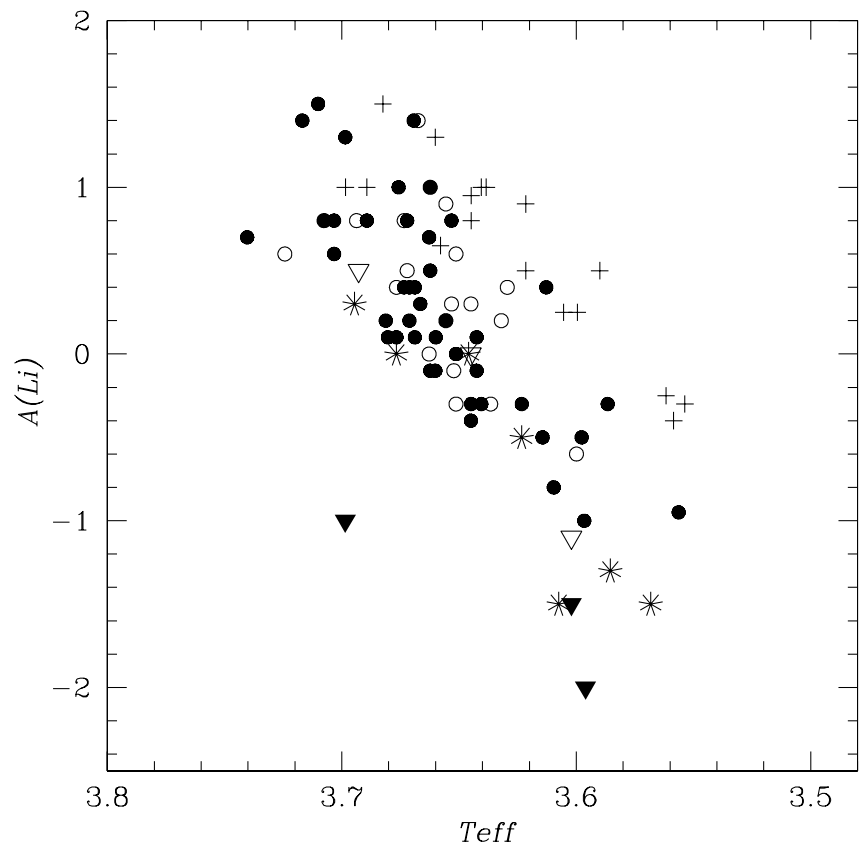

Fig. 1. $A_{\mathrm{Li}}$ as a function of effective temperature. Binary systems with eccentricity lower than about 0.10 are identified by open circles, whereas filled circles correspond to systems with an eccentricity higher than 0.10 . Filled inverted triangles are upper limits in the lithium abundance determination for systems with eccentricity higher than about 0.10 and open inverted triangles are upper limits in the lithium abundance determination for systems with an eccentricity lower than 0.10 . Stars from NGC 7789 and M 67 (Pilachowski et al. 1988) are identified by cross and asterisks, respectively.

\section{Working sample and observations}

The full sample analyzed in this study is composed by 68 binary systems with evolved component of luminosity class III, along the spectral region F, G and $\mathrm{K}$, with orbital period ranging from about 10 to about 6400 days. The stars were selected because of their bright apparent magnitude, orbital parameters available in the literature and measured CORAVEL rotational velocity. We emphasize, however, that the sample is not complete in any particular aspect and we should not have inserted any bias with respect to some physical parameter. The Li I observations were obtained at ESO, La Silla, in two observing runs, January 1997 and July 1998. The observations were carried out at the ESO CAT telescope equipped with the Coude Echelle Spectrometer. The covered spectral range was $56 \AA$ wide, the resolving power was 95000 , linear dispersion $1.83 \AA / \mathrm{mm}$ and signal-to-noise ratio between 80 and 200. Data reduction was performed by using the echelle context of the MIDAS package. The $A_{\mathrm{Li}}$ were determined using a LTE code derived from one kindly made available for us by Monique Spite, from the Paris-Meudon Observatory, following the same procedure described in de la Reza \& da Silva (1995), by fitting the synthetic spectra to the observed ones. In the computation of the synthetic spectra, all the known atomic lines in the range $\lambda 6702-$ $\lambda 6712$ were considered. The hyperfine structure of the

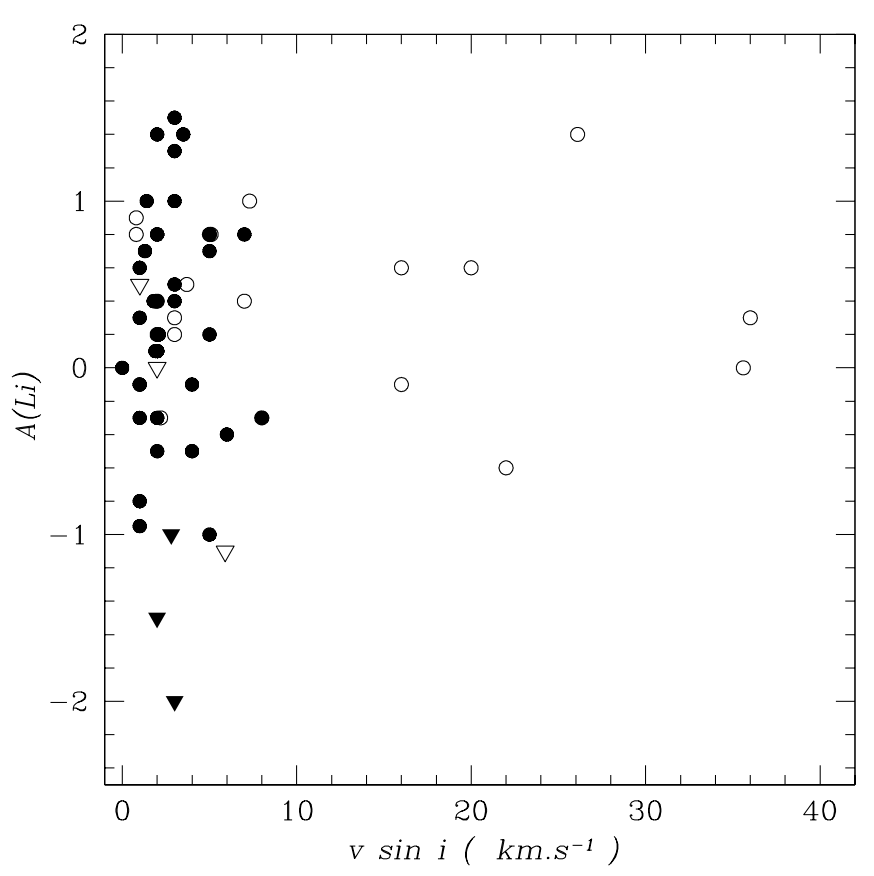

Fig. 2. $A_{\mathrm{Li}}$ as a function of rotational velocity. Open symbols represent binary systems with circularized orbit or nearly circularized orbit, namely those systems with eccentricity lower than about 0.10 , whereas the filled symbols stand for the systems with an eccentricity higher than 0.10 . Inverted triangles are for upper limits in the lithium abundance determination.

lithium resonance line was taken into account using the data quoted by Duncan (1991). The stellar atmospheric models used are from Gustafsson and collaborators (Bell et al. 1976). For all the stars we used the $\log g$ value given by the spectral type, microturbulence velocity of $2 \mathrm{~km} \mathrm{~s}^{-1}$ and solar abundances. Special care should be taken for the estimation of the effective temperature, since the derivation of $A_{\mathrm{Li}}$ is very sensitive to this parameter. For stars with the Stromgren ubvy photometry we have used the calibration from Moon (1985), where the effective temperature and surface gravity are given as a function of the $u b v y$ index. For those stars with no available Stromgren photometric index we have used the calibrations from Flower (1977) where the effective temperature is given as a function of the $(B-V)$ index and that from Pasquini et al. (1990) which gives the effective temperature as a function of the $(V-R)$ index. The derived lithium abundances as well as main stellar parameters and rotational velocities from CORAVEL, are presented in Table 1. Before discussing the results of the present study let us analyse how accurate are our lithium abundances and rotational velocities. First of all, we have compared the effective temperature values computed in the present work with those available in the literature for common stars, obtained by different authors. From 16 stars of Barrados et al. (1998), 6 stars of Brown et al. (1989) and 8 stars of Randich et al. (1994), in common with our sample, least square fits of the temperature values give, respectively, coefficients of correlation of about $0.90,0.96$ and 0.94 as well as rms of the differences of about $140 \mathrm{~K}, 104 \mathrm{~K}$ and $60 \mathrm{~K}$, respectively, 


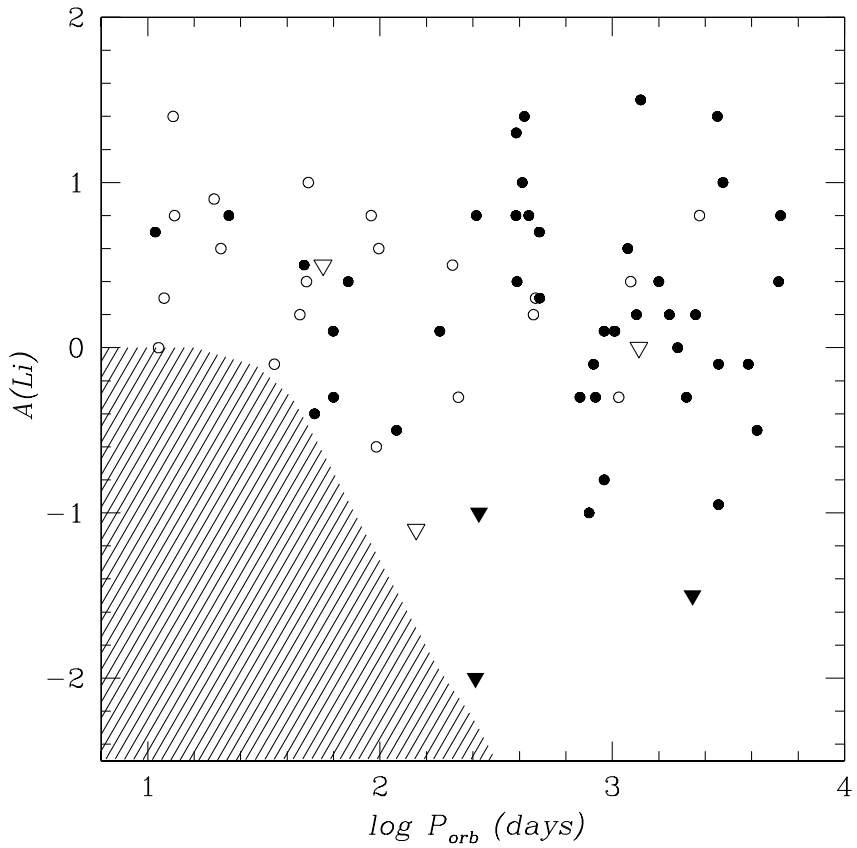

Fig. 3. $A_{\mathrm{Li}}$ as a function of the orbital period and eccentricity. Open symbols represent binary systems with circularized orbit or nearly circularized orbit, namely those systems with eccentricity lower than about 0.10 , whereas the filled symbols stand for the systems with an eccentricity higher than 0.10 . Inverted triangles are for upper limits in the lithium abundance and the shaded region defines a suggested "inhibited zone", as explained in the text.

indicating that our derived temperatures are in very reasonable agreement with others. Finally, we have compared Li abundances between the present study and the results of previous investigations cited above. For the 16 stars in common with Barrados et al. (1998) a least square fit gives a correlation coefficient of about 0.80. A comparison with the Li abundances from Brown et al. (1989) and Randich et al. (1994) gives a coefficient of correlation better than 0.80 .

\section{Results and discussion}

In Fig. 1 we show the behavior of the Li abundance for the binary systems with evolved component as a function of the effective temperature. The gradual decrease of lithium content with effective temperature, well established for single giants (e.g. Brown et al. 1989), is clearly observed for the binary systems. In addition, we are displaying in the Fig. 1 the $\mathrm{Li}$ abundances for the open clusters NGC 7789 and M 67 from Pilachowski et al. (1988), with ages of about 1.7 Gyrs and 5.0 Gyrs respectively. A comparison of the Li abundances of the present study with those for the cited open clusters shows, at a given effective temperature, a clear decrease of Li content with age for the binary systems with evolved component. Figure 2 presents the behavior of the $\mathrm{Li}$ abundances versus the projected rotational velocity $v \sin i$. Two interesting features emerge from this figure, paralleling the same link between rotation and lithium content for single giant stars (De Medeiros et al. 2000). First, one observes a large spread in the distribution of the values of $\mathrm{Li}$ abundance at low values of the rotational velocity. For binary systems with $v \sin i$ lower than about $5 \mathrm{~km} \mathrm{~s}^{-1}$, there is a spread of $\mathrm{Li}$ abundances from about -2 to about 1.5. Such a spread decreases with increasing rotation. Second, binary systems with moderate to high $v \sin i$ values, present also moderate to high values of $\mathrm{Li}$ abundance, this result pointing for a correlation between rotation and $\mathrm{Li}$ content. Figure 3 shows lithium abundance as a function of orbital period for binary systems with giant component. It is clear that for binary systems with an orbital period lower than about the expected period of synchronization, typically a period between 100 and 250 days, there is a strong inhibition in the lithium depletion. As indicated by the shaded area represented in the Fig. 3, such an "inhibited zone" is located below $A_{\mathrm{Li}}$ values around 0.0 and to the left of the expected values of the orbital period of synchronization. For the unsynchronized systems, namely those with an orbital period greater than about 100 to 250 days, one observes a large spread in $\mathrm{Li}$ abundances, with $A_{\mathrm{Li}}$ ranging from about -2 to about 1.5 , in contrast to synchronized systems which present a narrow spread in $A_{\mathrm{Li}}$ from about 0.0 to at least 1.5. Our finding is reinforced by the results of Barrado et al. (1998) for chromospherically active binary systems with giant component. By analyzing the lithium behavior of 66 such systems, most of them presenting synchronization features, with an orbital period lower than about 100 days, those authors have found no stars showing lithium abundance significantly below such a critical value of $A_{\mathrm{Li}}$ around 0.0, as represented in their Fig. 6b. Further, Barrado et al. (1998) list a few chromospherically active binary systems with giant component and orbital period shorter than 100 days showing $A_{\mathrm{Li}}$ around the cosmic value 3.0. Because the synchronization between rotational and orbital motion results from tidal effects, we claim that the "inhibited zone" is the result of the inhibition of the depletion of lithium due to such tidal effects. Hence, whereas synchronized binary systems have a tendency to retain more of their original lithium, the unsynchronized systems have normal lithium depletion. This explains the "inhibited zone", which indicates the absence of synchronized binary systems with giant component having a lithium abundance lower than a threshold level in the $A_{\mathrm{Li}}-P_{\text {orb }}$ plane.

\section{Conclusions}

Lithium abundances are presented for 68 binary systems with giant component. By combining these data with effective temperature, projected rotational velocity $v \sin i$ and orbital parameters, we show some very interesting trends on the behavior of Li content in this class of binary systems. The distribution of $\mathrm{Li}$ abundance as a function of effective temperature follows the same behavior observed for single giants, namely a gradual decrease of Li content with effective temperature. At low values of rotational 
Table 1. Lithium abundances, rotational velocities and orbital parameters for binary systems with evolved component.

\begin{tabular}{|c|c|c|c|c|c|c|c|}
\hline HD & $\mathrm{ST}$ & $B-V$ & $v \sin i$ & $A_{\mathrm{Li}}$ & $P_{\text {orb }}$ & $e$ & Ref \\
\hline 28 & K1III & 1.04 & 3.0 & 0.4 & 72.93 & 0.27 & $\mathrm{a}$ \\
\hline 352 & K2III & 1.38 & 22.0 & -0.6 & 96.439 & 0.04 & $\mathrm{a}$ \\
\hline 1833 & K1III & 1.13 & 16.3 & -0.1 & 35.100 & 0.04 & $\mathrm{~b}$ \\
\hline 2261 & KOIII & 1.09 & 1.0 & -0.1 & 3848.83 & 0.34 & $\mathrm{a}$ \\
\hline 7672 & G5IIIe & 0.90 & 1.0 & 0.5 & 56.8147 & 0.04 & $\mathrm{a}$ \\
\hline 12923 & KOIII & 0.90 & 2.0 & 0.8 & 5302 & 0.432 & $\mathrm{e}$ \\
\hline 19754 & G8III-IV & 1.12 & 7.0 & 0.4 & 48.263 & 0.1 & $\mathrm{c}$ \\
\hline 22905 & G8III & 0.88 & 0.8 & 0.8 & 91.629 & 0.0 & $\mathrm{a}$ \\
\hline 23817 & K2III & 1.13 & 0.0 & 0.0 & 1911.5 & 0.21 & $\mathrm{a}$ \\
\hline 34802 & K1IIIp & 1.09 & 0.8 & 0.9 & 19.310 & 0.0 & $\mathrm{~b}$ \\
\hline 37297 & G8-K0III & 0.83 & 2.0 & 0.1 & 180.87 & 0.51 & $\mathrm{a}$ \\
\hline 38099 & K4III & 1.47 & 5.9 & $<-1.1$ & 143.03 & 0.06 & $\mathrm{a}$ \\
\hline 43821 & G5III & 0.87 & 3.0 & 1.5 & 1325 & 0.44 & $\mathrm{a}$ \\
\hline 46407 & KOIII & 1.11 & 3.0 & 0.2 & 457.7 & 0.0 & $\mathrm{a}$ \\
\hline 49293 & K0IIIa & 1.11 & 2.0 & 0.2 & 1760.9 & 0.40 & $\mathrm{a}$ \\
\hline 50310 & K1III & 1.20 & 2.2 & -0.3 & 1066.0 & 0.09 & $\mathrm{a}$ \\
\hline 58972 & K3III & 1.22 & 1.8 & 0.4 & 389.0 & 0.31 & $\mathrm{a}$ \\
\hline 59717 & K5III & 1.52 & 3.0 & $<-2.0$ & 257.8 & 0.17 & $\mathrm{a}$ \\
\hline 61245 & K2III & 1.16 & 36.0 & 0.3 & 11.761 & 0.01 & $\mathrm{~b}$ \\
\hline 79910 & K2III & 1.17 & 2.0 & 0.1 & 922 & 0.29 & $\mathrm{a}$ \\
\hline 81410 & K1III & 1.02 & 26.1 & 1.4 & 12.8683 & 0.0 & $\mathrm{a}$ \\
\hline 82674 & KOIII & 1.17 & 1.0 & -0.1 & 830.4 & 0.15 & $\mathrm{a}$ \\
\hline 83240 & K1III & 1.05 & 2.0 & 1.4 & 2834 & 0.32 & $\mathrm{a}$ \\
\hline 83442 & K2IIIp & 1.16 & 6.0 & -0.4 & 52.270 & 0.13 & $\mathrm{~b}$ \\
\hline 88284 & KOIII & 1.01 & 1.9 & 0.4 & 1585.8 & 0.14 & $\mathrm{a}$ \\
\hline 92214 & G7.5III & 0.92 & 2.0 & 0.4 & 1200 & 0.1 & $\mathrm{a}$ \\
\hline 94363 & $\mathrm{KOIII+}$ & 0.90 & 1.0 & 0.6 & 1166 & 0.38 & $\mathrm{a}$ \\
\hline 102928 & K0III & 1.06 & 1.0 & 0.3 & 486.7 & 0.31 & $\mathrm{a}$ \\
\hline 112048 & KOIII & 1.09 & 1.9 & 0.1 & 1027 & 0.32 & $\mathrm{a}$ \\
\hline 112985 & K2III & 1.18 & 1.0 & -0.3 & 847 & 0.4 & $\mathrm{a}$ \\
\hline 119834 & G9III & 0.96 & 2.0 & 0.8 & 437.00 & 0.13 & $\mathrm{a}$ \\
\hline 120901 & KOIII & 1.08 & 5.0 & 0.2 & 2283.0586 & 0.49 & $\mathrm{a}$ \\
\hline 133461 & K2III & 1.16 & 2.0 & -0.3 & 725.5 & 0.29 & $\mathrm{a}$ \\
\hline 136905 & K1III & 1.03 & 35.6 & 0.0 & 11.1345 & 0.00 & $\mathrm{a}$ \\
\hline 139137 & G8III & 0.72 & 2.0 & 0.8 & 259.81 & 0.378 & $\mathrm{f}$ \\
\hline 145206 & K4III & 1.45 & 2.0 & -0.3 & 2084.8 & 0.55 & $\mathrm{a}$ \\
\hline 147508 & K2III & 1.33 & 1.0 & $<-0.8$ & 922.8 & 0.37 & $\mathrm{a}$ \\
\hline 156731 & K3III & 1.45 & 5.0 & -1.0 & 794.5 & 0.69 & $\mathrm{a}$ \\
\hline 158837 & G8III & 0.84 & 3.5 & 1.4 & 418.242 & 0.20 & $\mathrm{a}$ \\
\hline 162391 & G8III & 1.13 & 8.0 & -0.3 & 217.440 & 0.0 & $\mathrm{~d}$ \\
\hline 162596 & KOIII & 1.12 & 3.0 & 0.3 & 467.2 & 0.0 & $\mathrm{a}$ \\
\hline 165141 & G8-K0III & 1.01 & 2.0 & 0.4 & 5200. & $\ldots$ & $\mathrm{c}$ \\
\hline 168339 & K4III & 1.48 & 2.0 & $<-1.5$ & 2214 & 0.26 & $\mathrm{a}$ \\
\hline 169156 & G9IIIb & 0.94 & 5.0 & 0.8 & 2373.7911 & 0.10 & $\mathrm{a}$ \\
\hline 169689 & G8III-IV & 0.92 & 7.0 & 0.8 & 385 & 0.31 & $\mathrm{a}$ \\
\hline 169985 & G0III & 0.50 & 3.0 & 1.3 & 386.0 & 0.47 & $\mathrm{a}$ \\
\hline 172831 & K0-1III & 1.00 & 1.3 & 0.7 & 485.3 & 0.21 & $\mathrm{a}$ \\
\hline 175515 & K0III & 1.04 & 1.4 & 1.0 & 2994 & 0.24 & $\mathrm{a}$ \\
\hline 176411 & K1III & 1.08 & 2.1 & 0.2 & 1270.6 & 0.27 & $\mathrm{a}$ \\
\hline 178717 & K4III & 1.88 & 1.0 & -0.95 & 2866 & 0.434 & $\mathrm{~g}$ \\
\hline 179950 & F2:+F1III & 0.55 & 5.0 & 0.7 & 10.7786 & 0.47 & $\mathrm{a}$ \\
\hline 181391 & G8III-IV & 0.92 & 2.8 & $<-1.0$ & 266.544 & 0.83 & $\mathrm{a}$ \\
\hline 181809 & K2III & 1.17 & 5.1 & 0.8 & 13.048 & 0.05 & $\mathrm{c}$ \\
\hline 182776 & K2/K3III & 1.17 & 2.0 & 0.2 & 45.180 & 0.02 & $\mathrm{c}$ \\
\hline 185510 & KOIII-IV & 0.84 & 16.0 & 0.6 & 20.660 & 0.10 & $\mathrm{~b}$ \\
\hline
\end{tabular}


Table 1. continued

\begin{tabular}{cccrrrrr}
\hline HD & ST & $B-V$ & $v \sin i$ & $A_{\mathrm{Li}}$ & \multicolumn{1}{c}{$P_{\text {orb }}$} & $e$ & Ref \\
\hline & & & & & & & \\
188981 & K1III & 1.05 & 2.0 & 0.1 & 62.877 & 0.34 & $\mathrm{a}$ \\
194184 & K3III & 1.36 & 4.0 & -0.5 & 117.776 & 0.24 & $\mathrm{a}$ \\
196574 & G8III & 0.95 & 3.7 & 0.5 & 205.2 & 0.0 & $\mathrm{a}$ \\
202134 & K1IIIp & 1.12 & 8.0 & -0.3 & 63.09 & 0.52 & $\mathrm{c}$ \\
204128 & K1III & 1.12 & 5.0 & 0.8 & 22.349 & 0.12 & $\mathrm{c}$ \\
202447 & G0III+ & 0.53 & 20.0 & 0.6 & 98.81 & 0.04 & $\mathrm{a}$ \\
205249 & K1IIIp & 1.08 & 7.3 & 1.0 & 49.137 & 0.08 & $\mathrm{c}$ \\
205478 & K0III & 1.00 & 2.0 & 0.1 & 1020 & 0.4 & $\mathrm{a}$ \\
211416 & K3III & 1.39 & 2.0 & -0.5 & 4197.7 & 0.39 & $\mathrm{a}$ \\
213428 & K0III & 1.08 & 4.0 & -0.1 & 2866 & 0.5 & $\mathrm{a}$ \\
217188 & K0III & 1.08 & 3.0 & 0.5 & 47.121 & 0.50 & $\mathrm{c}$ \\
218670 & K1III & 1.02 & 3.0 & 1.0 & 409.614 & 0.66 & $\mathrm{a}$ \\
223617 & G9III & 1.16 & 2.0 & $<0.0$ & 1301 & 0.098 & $\mathrm{~g}$ \\
& & & & & & &
\end{tabular}

${ }^{\text {a }}$ Batten et al. (1989); ${ }^{\mathrm{b}}$ Strassmeier et al. (1988); ${ }^{\mathrm{c}}$ Strassmeier et al. (1993); ${ }^{\mathrm{d}}$ Mermilliod et al. (1989); ${ }^{\mathrm{e}}$ Griffin (1989);

${ }^{\mathrm{f}}$ Griffin (1990); ${ }^{\mathrm{g}}$ McClure et al. (1990).

velocity, typically for $v \sin i$ lower than about $5 \mathrm{~km} \mathrm{~s}^{-1}$, there is a large spread in the values of $\mathrm{Li}$ abundance of at least 3.5 magnitudes. Such a spread decreases for increasing rotation, following the same trend observed for their single counterparts. In spite of this large spread in Li abundances for the slow rotators, we observe that the moderate to high rotators present a tendency for moderate to high lithium contents, this result pointing for a correlation between rotation and Li content. Finally, the analysis of the Li abundance as a function of orbital period seems to show the effect of tidal interaction on the dilution of lithium. Binary systems with orbital period lower than about 100 days, typically those systems showing synchronization between rotational and orbital motions, present $\mathrm{Li}$ abundance enhanced in relation to the systems with orbital period larger than 100 days. In fact, in the $A_{\mathrm{Li}}-$ $P_{\text {orb }}$ plane it seems to exist an "inhibited zone" where binary systems with giant component showing synchronization between rotational and orbital motions and abundances lower than a threshold value around 0.0 appear to be unusual.

Acknowledgements. This work has been supported by continuous grants from the CNPq Brazilian Agency. L. da S. thanks the CNPq for financial support through grant 200580/970. J.D.N.Jr. acknowledges the CNPq grant 300925/99-9. We would like to thank the referee for his useful comments and suggestions on the manuscript.

\section{References}

Barrado y Navascues, D., de Castro, E., Fernandez-Figueroa, M. J., Cornide, M., \& Garcia López, R. J. 1998, A\&A, 337, 739

Batten, A. H., Fletcher, J. M., \& MacCarthy, D. G. 1989, Publ. Dom. Astrophys. Obs., 17, 1

Bell, R. A., Eriksson, K., Gustafsson, B., \& Nordlund, A. 1976, A\&AS, 23, 37
Brown, J. A., Sneden, C., Lambert, D. L., \& Dutchover Jr., E. 1989, ApJS, 71, 293

De Medeiros, J. R., do Nascimento Jr., J. D., \& Mayor, M. 1997, A\&A, 317, 701

De Medeiros, J. R., do Nascimento Jr., J. D., Sankarankutty, S., Costa, J. M., \& Maia, M. R. G. 2000, A\&A, 363, 239

de la Reza, R., \& da Silva, L. 1995, ApJ, 439, 917

do Nascimento Jr., J. D., Charbonnel, C., Lèbre, A., de Laverny, P., \& De Medeiros, J. R. 2000, A\&A, 357, 931

Duncan, D. K. 1991, ApJ, 373, 250

Flower, P. J. 1977, A\&A, 54, 31

Garcia Lopez, R. J., Rebolo, R., \& Martin, E. L. 1994, A\&A, 282,518

Griffin, R. F. 1989, The Observatory, 109, 142

Griffin, R. F. 1990, JApA, 11, 491

McClure, R. D., \& Woodsworth, A. W. 1990, ApJ, 352, 709

Moon, T. 1985, Comm. University of London Observatory, No. 78

Mermilliod, J.-C., Mayor, M., Andersen, J., et al. 1989, A\&AS, 79,11

Pasquini, L., Brocato, E., \& Pallavicini, R. 1990, A\&A, 234, 277

Pilachowski, C. A., Saha, A., \& Hobbs, L. M. 1988, PASP, 100, 474

Pinsonneault, M. H., Kawaler, S. D., Sofia, S., \& Demarque, P. 1989, ApJ, 338, 424

Pinsonneault, M. H., Kawaler, S. D., \& Demarque, P. 1990, ApJS, 74, 501

Randich, S., Giampapa, M. S., \& Pallavicini, R. 1994, A\&A, 283,893

Randich, S., Martin, E. L., Garcia López, R. J., \& Pallavicini, R. 1998, A\&A, 333, 591

Randich, S., Gratton, R., Pallavicini, R., Pasquini, L., \& Carretta, E. 1999, A\&A, 348, 487

Spite, M., Pasquini, L., \& Spite, F. 1994, A\&A, 290, 217

Strassmeier, K. G., Hall, D. S., Zeilik, M., et al. 1988, A\&AS, 72,291

Strassmeier, K. G., Hall, D. S., Fekel, F. C., \& Scheck, M. 1993, A\&AS, 100, 173

Zahn, J. P. 1977, A\&A, 57, 383

Zahn, J. P. 1994, A\&A, 288, 829 